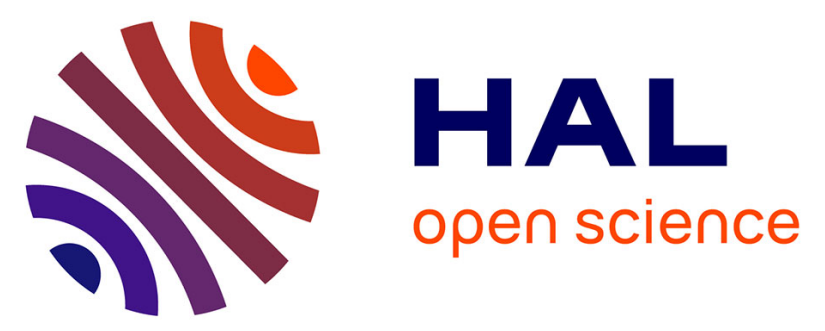

\title{
Cycle Slip Detection and Repair Using an Array of Receivers with Known Geometry for RTK Positioning
}

Xiao Hu, Paul Thevenon, Christophe Macabiau

\section{To cite this version:}

Xiao Hu, Paul Thevenon, Christophe Macabiau. Cycle Slip Detection and Repair Using an Array of Receivers with Known Geometry for RTK Positioning. PLANS 2020 IEEE/ION Position, Location and Navigation Symposium, Apr 2020, Portland, United States. pp.1123-1134 / ISBN: 978-1-72819446-2, 10.1109/PLANS46316.2020.9109871 . hal-02866389

\section{HAL Id: hal-02866389 \\ https://hal-enac.archives-ouvertes.fr/hal-02866389}

Submitted on 15 Jun 2020

HAL is a multi-disciplinary open access archive for the deposit and dissemination of scientific research documents, whether they are published or not. The documents may come from teaching and research institutions in France or abroad, or from public or private research centers.
L'archive ouverte pluridisciplinaire HAL, est destinée au dépôt et à la diffusion de documents scientifiques de niveau recherche, publiés ou non, émanant des établissements d'enseignement et de recherche français ou étrangers, des laboratoires publics ou privés. 


\title{
Cycle Slip Detection and Repair Using an Array of Receivers with Known Geometry for RTK Positioning
}

\author{
Xiao Hu, Paul Thevenon, Christophe Macabiau \\ ENAC, Université de Toulouse \\ Toulouse, FRANCE \\ xiao.hu@recherche.enac.fr
}

\begin{abstract}
Currently, precise position information is a certain requirement for many application scenarios. The GNSS carrier phase measurements appear mandatory to satisfy the sub-meter or even centimeter-level for these high precision navigation and positioning requirements. To achieve this level of accuracy, the ambiguity of carrier phase measurements must be correctly resolved and maintained, and the cycle slip must be correctly repaired. In this paper, we firstly use multiple receivers with known geometry to ameliorate the performance of the RTK positioning and cycle slip detection and repair process in different environments by taking advantage of the doubled number of observations. We demonstrate through different simulated scenarios that we can detect the occurrence of cycle slip more accurately and timely. The simulations results show that our multi-receiver RTK system is more robust to cycle slips with a higher rate of occurrence or lower size, and also to harsh measurement noise, in terms of ambiguity fixing rate. Overall, better positioning accuracy is obtained under the same conditions when comparing with the single receiver system.
\end{abstract}

Keywords-Cycle Slip Detection and Repair, Real-Time Kinematic (RTK), Integer Ambiguity Resolution, Precise Positioning, GNSS

\section{INTRODUCTION}

The concept of autonomous driving has become more and more a central topic for the automobile industry, where a precise position and attitude information is essential for this kind of application. Over the past decade, the universal GNSS has been dramatically utilized in various domains, such as aviation, marine, precise agriculture, geodesy, and surveying, automotive, etc. However, the accuracy or integrity that a lowcost GNSS receiver can provide in a constrained urban or indoor environment is far from satisfactory for applications where decimeter or centimeter accuracy and error bounds are mostly envisioned. To reach this level of accuracy, techniques using raw carrier phase measurements have been developed. Carrier phase measurements are more precise than code measurements by a factor of a hundred. Nevertheless, they are also less robust and include a so-called integer ambiguity (IA) that prevents them to be used directly for positioning. In some harsh environments, severe code multipath, frequent Cycle Slips (CS), and losses of lock might also challenge the process of finding the correct IA (Integer Ambiguity Resolution, abbreviated IAR) thus reduce the accuracy.
In order to increase confidence and speed up the process of finding the correct ambiguity integers, constraints can be established by using an array of two or more receivers with prior known and fixed geometry, including the length of the baseline vectors between the receivers of the array and the orientation of the vectors to limit the search space.

Recently, several studies have focused on the use of an array of receivers for attitude determination [1], [2] and calibration of magnetic field sensors [3], Daniel et al. [4] developed a method for the recursive estimation of the positioning and attitude problems using GNSS carrier phase observations from an array of receivers, but they calculated the position of each receiver separately thus they didn't take advantage of the known geometry. Farhad et al. [5] [6] used an adaptive KF for 3dimensional attitude determination and position estimation of a mobile robot by fusing the information from a system of two RTK GPSs and an IMU, however, they also did not consider the known geometry of the receiver as a constraint to help improve the performance. Nandakumaran et al. [7] provided a numerical insight into the role taken by the multi-GNSS integration in delivering high-precision solutions, however, they focused on the PPP solution rather than the RTK processing which requires long convergence time to reach cm-level accuracy. Khodabandeh et al. [8], [9] introduced a concept of array-based between-satellite single difference satellite phase biases determination to accelerate the single-receiver IAR, but they did not take into account the attitude information of the vehicle.

As far as we know, the use of an array of receivers with attitude determination is not used to improve the accuracy of the array position or for some internal steps of precise position computation, such as Cycle Slip Detection and Repair (CS-DR) or integer ambiguity resolution. The concept of RTK positioning using an array of GNSS receiver has already been introduced in [10]. In this work, we will focus on the improvement of cycleslip detection and repair for RTK processing by using an array of two receivers with known geometry.

\section{MATHEMATICAL MODELS}

\section{A. The geometry of the Array Receivers Model}

In this study, we propose an Extended Kalman Filter (EKF) based position and attitude determination algorithm. In order to perform precise attitude estimation, a dual antenna set-up has been considered, where two GNSS antennas with a known 
baseline length are mounted on the vehicle's rooftop to get an attitude estimation. Furthermore, the absolute position accuracy is augmented using real-time kinematics (RTK) approach, in which the vehicle is positioned relative to a third receiver as the virtual reference station (VRS), whose position is assumed static and known. By knowing the absolute position of the VRS, the vehicle can be positioned absolutely, too. In the positioning algorithm, we strongly rely on carrier phase positioning which, thanks to its low noise characteristics, may enable centimeterlevel positioning. Figure 1 shows the typical geometry of our measurement set-up.

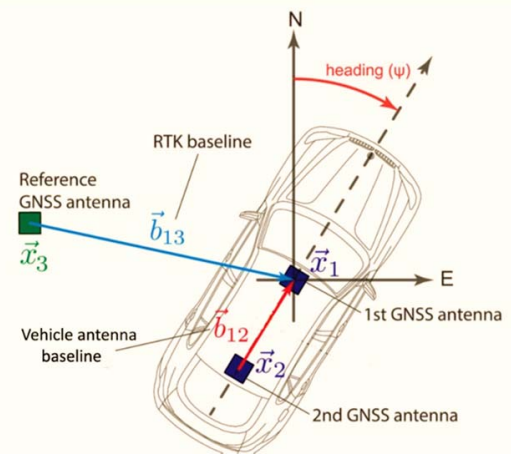

Fig. 1. The geometry of the model [10]

We estimate both the RTK position and receiver array attitude for every time epoch, the former being defined as a function of the absolute position $\vec{x}_{1}$ and $\vec{x}_{3}$ :

$$
\vec{b}_{13}=\vec{x}_{1}-\vec{x}_{3}
$$

With the latter being defined as a function of Euler attitude angles with the vehicle antenna baseline length $l=\left\|\vec{b}_{12}\right\|$, the heading $\psi$ and the pitch $\theta$ of the vehicle. The vehicle antenna array baseline vector $\vec{b}_{12}$ has the following coordinates in the local navigation (East, North, Up) ENU frame:

$$
\vec{b}_{12}(\psi, \theta)=\left\|\vec{b}_{12}\right\| \cdot\left[\begin{array}{c}
\cos (\theta) \sin (\psi) \\
\cos (\theta) \cos (\psi) \\
\sin (\theta)
\end{array}\right]
$$

It should be noted that the absolute position $\vec{x}_{3}$ of the reference GNSS antenna is static and known and the norm $\left\|\vec{b}_{12}\right\|$ of the vehicle antenna baseline is always constant and known a priori, too. Thus, the heading and pitch angle estimates follow from the fixed baseline estimate by simple trigonometric properties as

$$
\begin{gathered}
\hat{\psi}\left(t_{i}\right)=\operatorname{atan}\left(\frac{\left(\vec{b}_{12}\right)_{x}\left(t_{i}\right)}{\left(\vec{b}_{12}\right)_{y}\left(t_{i}\right)}\right) \\
\hat{\theta}\left(t_{i}\right)=\operatorname{atan}\left(\frac{\left(\vec{b}_{12}\right)_{z}\left(t_{i}\right)}{\sqrt{\left(\vec{b}_{12}\right)_{x}^{2}\left(t_{i}\right)+\left(\vec{b}_{12}\right)_{y}^{2}\left(t_{i}\right)}}\right)
\end{gathered}
$$

In the following, the state and the measurement vectors are described along with the state-transition and measurement models.

\section{B. State Transition Model}

The state transition model describes how the states or parameters of the system vary over time based on a specific linear model. In our EKF modeling, the state parameter transition between subsequent epochs is given by:

Where:

$$
\mathbf{x}_{n}=\boldsymbol{\phi}_{n-1} \mathbf{x}_{n-1}+\mathbf{w}_{n}
$$

- $\mathbf{x}_{n}$ refers to the state vector at epoch $n$

- $\boldsymbol{\phi}_{n-1}$ refers to the transition matrix from epoch $n-1$ to epoch $n$

- $\mathbf{w}_{n}$ refers to the so-called system noise vector at epoch $n$

Together with the process noise vector, one can define the process noise covariance matrix as:

$$
\mathbf{Q}_{n}=E\left[\mathbf{w}_{n} \mathbf{w}_{n}^{\mathrm{T}}\right]
$$

This matrix has then the variances of the state parameter's estimates based on the system model.

The estimated parameters are collected inside the state vector. The state vector collects 5 vehicle state parameters and $2 *\left(N_{s a t}-1\right)$ satellite state parameters which are: the $3 \mathrm{D}$ position of GNSS receiver 1 relative to GNSS receiver 3 , heading angle of the vehicle, pitch angle of the vehicle, double-difference integer ambiguity of the GNSS receiver pair 1-3 and the doubledifference integer ambiguity of the receiver pair $2-3$. which is given by:

where:

$$
\mathbf{x}=\left[\begin{array}{lllll}
\mathbf{b}_{13}^{T} & \theta & \psi & \mathbf{N}_{13}^{T} & \mathbf{N}_{23}^{T}
\end{array}\right]^{T}
$$

- $\quad \boldsymbol{N}_{n p}^{T}$ refers to the vector of the double-difference GPS integer ambiguity between receiver $n$ and $p$

In our EKF modeling, for the position and attitude related state parameters, we suppose that it is a random walk model, the speed and the angular rate are a zero-mean Gaussian process.

$$
\begin{aligned}
\mathbf{b}_{13, n} & =\mathbf{b}_{13, n-1}+\mathbf{w}_{13, n} \\
\theta_{n} & =\theta_{n-1}+w_{\theta, n} \\
\psi_{n} & =\psi_{n-1}+w_{\psi, n}
\end{aligned}
$$

Where:

- $\quad \mathbf{w}_{13}$ is a centered Gaussian vector with a covariance matrix $\mathbf{Q}_{13}$

- $w_{\theta}$ is a centered Gaussian variable with a standard deviation $\sigma_{\theta}$

- $w_{\psi}$ is a centered Gaussian variable with a standard deviation $\sigma_{\psi}$

In the case of the satellite-related parameters, they are assumed all as constant over subsequent epochs with a very small noise compared to the position and attitude related state parameters.

$$
\begin{aligned}
& \mathbf{N}_{13, n}=\mathbf{N}_{13, n-1}+\mathbf{w}_{a m b, n} \\
& \mathbf{N}_{23, n}=\mathbf{N}_{23, n-1}+\mathbf{w}_{a m b, n}
\end{aligned}
$$


where:

- $\mathbf{w}_{a m b, n}$ is a centered Gaussian vector with a covariance matrix $\mathbf{Q}_{a m b}=\sigma_{a m b}^{2} \mathbf{I}_{N_{s a t}-1}$

- $\mathbf{I}_{N}$ is the identity matrix of size $N$

The resulting state transition matrix $\boldsymbol{\phi}$ is then given by a unit matrix and different values of process noise variance are added to complete the model

$$
\boldsymbol{\Phi}=\left[\begin{array}{ccc}
1 & \cdots & 0 \\
\vdots & \ddots & \vdots \\
0 & \cdots & 1
\end{array}\right]
$$

And the corresponding process noise matrix $\mathbf{Q}$ is given as follows:

$$
\mathbf{Q}=\left[\begin{array}{ccccc}
\mathbf{Q}_{13} & \mathbf{0}_{3 \times 1} & \mathbf{0}_{3 \times 1} & \mathbf{0}_{3 \times N_{s a t}-1} & \mathbf{0}_{3 \times N_{s a t}-1} \\
\mathbf{0}_{1 \times 3} & \sigma_{\theta}^{2} & 0 & \mathbf{0}_{1 \times N_{s a t}-1} & \mathbf{0}_{1 \times N_{s a t}-1} \\
\mathbf{0}_{1 \times 3} & 0 & \sigma_{\psi}^{2} & \mathbf{0}_{1 \times N_{s a t}-1} & \mathbf{0}_{1 \times N_{s a t}-1} \\
\mathbf{0}_{N_{s a t}-1 \times 3} & \mathbf{0}_{N_{s a t}-1 \times 1} & \mathbf{0}_{N_{s a t}-1 \times 1} & \mathbf{Q}_{a m b} & \mathbf{0}_{N_{s a t}-1} \\
\mathbf{0}_{N_{s a t}-1 \times 3} & \mathbf{0}_{N_{s a t}-1 \times 1} & \mathbf{0}_{N_{s a t}-1 \times 1} & \mathbf{0}_{N_{s a t}-1} & \mathbf{Q}_{a m b}
\end{array}\right]
$$

\section{Measurement Model}

The measurement model describes how the individual sensor measurements are related to system states. In general, for every epoch $\mathrm{n}$, the measurement vector $\mathbf{z}_{\boldsymbol{n}}$, which contains all measured values, can be described as a function of the state vector $\mathbf{x}_{n}$ as:

$$
\mathbf{z}_{n}=\mathbf{h}_{n}\left(\mathbf{x}_{n}\right)+\mathbf{v}_{n}
$$

with $\mathbf{h}_{n}$ the function that relates one or more states with each measured value and $\mathbf{v}_{n}$ the measurement noise vector, which describes the expected Gaussian noise of every measured value. As we are modeling an extended Kalman filter, $\mathbf{h}_{n}$ is a usually non-linear function.

As for the process noise covariance matrix, the definition of the measurement noise covariance matrix follows as:

$$
\mathbf{R}_{n}=E\left[\mathbf{v}_{n} \mathbf{v}_{n}^{\mathrm{T}}\right]
$$

In our model, the measurement vector comprises the following measured values: Double-difference (DD) code phase measurement vector of receiver 1, DD code phase measurement vector of receiver 2, DD carrier phase measurement vector of receiver 1, and DD carrier phase measurement vector of receiver 2.

$$
\mathbf{z}_{n}=\left[\begin{array}{llll}
\left(\mathbf{P}_{13}\right)^{T} & \left(\mathbf{P}_{23}\right)^{T} & \lambda\left(\boldsymbol{\phi}_{13}\right)^{T} & \lambda\left(\boldsymbol{\phi}_{23}\right)^{T}
\end{array}\right]^{T}
$$

In this measurement model, the position of receiver 2 is expressed in terms of the position of receiver 1 and the baseline vector between the 2 receivers of the array, such that it contains the known array baseline length information and the attitude information that we want to estimate. The individual doubledifference corrected pseudo-range and phase GPS measurement for our short baseline case (less than $1 \mathrm{~km}$ ) can be modeled as:

$$
\begin{gathered}
P_{13}^{k l}=\left(\mathbf{e}^{k l}\right)^{T} \mathbf{b}_{13}+n_{P, 13}^{k l} \\
P_{23}^{k l}=\left(\mathbf{e}^{k l}\right)^{T}\left(\mathbf{b}_{13}-l\left[\begin{array}{c}
\cos \theta \sin \psi \\
\cos \theta \cos \psi \\
\sin \theta
\end{array}\right]\right)+n_{P, 23}^{k l}
\end{gathered}
$$

$$
\begin{gathered}
\lambda \phi_{13}^{k l}=\left(\mathbf{e}^{k l}\right)^{T} \mathbf{b}_{13}+\lambda N_{13}^{k l}+n_{\phi, 13}^{k l} \\
\lambda \phi_{23}^{k l}==\left(\mathbf{e}^{k l}\right)^{T}\left(\mathbf{b}_{13}-l\left[\begin{array}{c}
\cos \theta \sin \psi \\
\cos \theta \cos \psi \\
\sin \theta
\end{array}\right]\right)+\lambda N_{23}^{k l}+n_{\phi, 23}^{k l}
\end{gathered}
$$

Where:

- $P_{n p}^{k l} \quad$ refers to the double-difference code phase measurement vector of receiver pair $n p$ and satellite pair $k l$

- $\lambda \phi_{n p}^{k l}$ refers to the carrier phase measurement vector of receiver pair $n p$ and satellite pair $k l$

- $\mathbf{e}^{k l}$ refers to the difference between the Line of Sight vector of satellite $k$ and $l$

- $\quad N_{n p}^{k l}$ refers to the double-difference integer ambiguity of receiver pair $n p$ and satellite pair $k l$

- $\quad n_{P, n p}^{k l}, n_{\phi, n p}^{k l}$ refers to the noise measurement of the double-difference code and phase measurement respectively

Additionally, the LOS vectors are modeled by using the azimuth $\varphi_{k, r}$ and elevation $\theta_{k, r}$ of the satellites with respect to the corresponding receiver, thanks to the following formula who define the LOS (Line Of Sight) vector between satellite $k$ and receiver $r$ in the ENU frame:

$$
\mathbf{e}_{r}^{k}=\left[\begin{array}{c}
\cos \theta_{k, r} \cos \varphi_{k, r} \\
\cos \theta_{k, r} \sin \varphi_{k, r} \\
\sin \theta_{k, r}
\end{array}\right]
$$

To reflect the difference in accuracy between the code measurement and the carrier phase measurement, a fixed scale factor is applied:

Where:

$$
\sigma_{\text {phase }}=a * \sigma_{\text {code }}
$$

- $\sigma_{\text {code }}$ refers to the standard deviation of code measurement

- $\sigma_{\text {phase }}$ refers to the standard deviation of carrier phase measurement

- $a$ refers to the ratio between $\sigma_{\text {code }}$ and $\sigma_{\text {carrier }}$ to account for the much better accuracy of carrier phase measurements

A fixed measurement noise variance between all satellites is defined to complete the measurement model, defining the measurement covariance matrix R. Firstly, the measurement covariance matrix $\mathbf{R}_{\mathrm{SD}}$ for the single difference measurements will have the following shape:

$$
\begin{aligned}
\mathbf{R}_{\text {code }, \mathrm{SD}} & =2 * \sigma_{\text {code }}^{2}\left[\begin{array}{ll}
\mathbf{I}_{N_{\text {sat }}} & \mathbf{0}_{\text {Nsat }} \\
\mathbf{0}_{\text {Nsat }} & \mathbf{I}_{N_{\text {sat }}}
\end{array}\right], \\
\mathbf{R}_{\text {carrier }, \mathrm{SD}} & =2 * a^{2} * \sigma_{\text {code }}^{2}\left[\begin{array}{ll}
\mathbf{I}_{N_{\text {sat }}} & \mathbf{0}_{\text {Nsat }} \\
\mathbf{0}_{\text {Nsat }} & \mathbf{I}_{N_{\text {sat }}}
\end{array}\right]
\end{aligned}
$$

The measurement covariance matrix $\mathbf{R}$ for the doubledifference measurements can then be deduced using the following formula: 
Where :

$$
\mathbf{R}=\mathbf{D}\left[\begin{array}{cc}
\mathbf{R}_{\text {code }, \mathrm{SD}} & \mathbf{0}_{N_{\text {sat }}} \\
\mathbf{0}_{N_{\text {sat }}} & \mathbf{R}_{\text {carrier }, \mathrm{SD}}
\end{array}\right] \mathbf{D}^{\boldsymbol{T}}
$$

$$
\boldsymbol{D}=\left[\begin{array}{ccccc}
-1 & 0 & \cdots & 0 & 1 \\
0 & -1 & \ddots & \vdots & 1 \\
\vdots & \ddots & \ddots & 0 & \vdots \\
0 & \cdots & 0 & -1 & 1
\end{array}\right]
$$

is the single-differencing matrix used for computing the doubledifference assuming the last satellite is used as a pivot for the double-difference computation.

The relationship between the state and measurement vector is obviously not linear thus we need the EKF to help linearize the non-linear measurement function to the measurement matrix H.

Two alternating steps which are the state prediction step and the state update step are then conducted to complete the algorithm.

\section{EXTENDED KALMAN FILTER PROCESSING}

The Kalman Filter algorithm is an iterative algorithm that comprises basically two alternating steps: the state prediction step and the state update step. In the first one, the state transition model is applied to predict the behavior of the system in the next epoch basing on a-priori information such as those coming from the movement model. In the second step, the update step, the prediction is confronted with the actual measurements, and a trade-off between the two estimates is chosen as optimal. This optimum is computed based on the stochastic properties both state transition and measurement model with a Bayesian approach on a MMSE (minimum mean square error) basis.

\section{A. The prediction step}

In the prediction step, the system model is used to estimate the state variable's value for the subsequent epoch. In this phase, a prediction on the state parameter's values on the subsequent epoch is done only by assuming a linear model, such as a movement model. The equation that describes this prediction uses the section State transition model excluding the state noise component.

Where:

$$
\hat{\mathbf{x}}_{n}^{-}=\boldsymbol{\Phi}_{n-1} \hat{\mathbf{x}}_{n-1}^{+}
$$

- $\quad \hat{\mathbf{x}}_{n-1}^{+}$refers to the estimate of the state vector coming from the last update phase epoch n-1

- $\hat{\mathbf{x}}_{n}^{-}$refers to the estimate of the predicted state vector for the current epoch $n$

Together with the state vector, there is also another quantity that should be updated: namely the state covariance matrix, which is defined as the expected value of the state vector residuals, which in turn are defined as the difference or error between the real and estimated state vector:

$$
\mathbf{P}=\mathbf{E}\left[(\mathbf{x}-\hat{\mathbf{x}})(\mathbf{x}-\hat{\mathbf{x}})^{\mathrm{T}}\right]
$$

where $\mathbf{x}$ is the true and $\hat{\mathbf{x}}$ is the estimated state vector. This matrix could be related either to the prediction or update step, in which case the matrix is called a-priori or a-posteriori state covariance matrix.

In the prediction step, the a-priori state covariance matrix $\mathbf{P}_{n}^{-}$is updated as:

$$
\mathbf{P}_{n}^{-}=\boldsymbol{\Phi}_{n-1} \mathbf{P}_{n-1}^{+} \boldsymbol{\Phi}_{n-1}^{\mathrm{T}}+\mathbf{Q}_{n-1}
$$

where $\mathbf{P}_{n-1}^{+}$represents the a-posteriori state covariance matrix of the previous state update step.

\section{B. The update step}

In this step, the measured values are taken into account and "fed back" to the system, the relationship between the state and measurement vector has been mentioned in the Measurement model section:

$$
\mathbf{z}_{n}=\mathbf{h}_{n}\left(\mathbf{x}_{n}\right)+\mathbf{v}_{n}
$$

The non-linear measurement function $\mathbf{h}_{n}\left(\mathbf{x}_{n}\right)$ is linearized to the measurement matrix $\mathbf{H}_{n}$ as:

$$
\mathbf{h}_{n}\left(\mathbf{x}_{n}\right) \approx \mathbf{H}_{n} \mathbf{x}_{n}=\mathbf{h}_{n}\left(\hat{\mathbf{x}}_{n}^{-}\right)+\mathbf{H}_{n}\left(\mathbf{x}_{n}-\hat{\mathbf{x}}_{n}^{-}\right)
$$

which is then used to calculate the Kalman gain matrix $\mathbf{K}_{n}$ and the a-posteriori covariance matrix $\mathbf{P}_{n}^{+}$, as will be seen later in this section. The calculation of the measurement matrix can be then performed as:

In our case:

$$
\left.\mathbf{H}_{n}\right|_{\mathbf{x}_{n}=\hat{\mathbf{x}}_{n}^{-}}=\left.\frac{\partial}{\partial \mathbf{x}_{n}} \mathbf{h}_{n}\left(\mathbf{x}_{n}\right)\right|_{\mathbf{x}_{n}=\hat{\mathbf{x}}_{n}^{-}}
$$

$$
\frac{\partial P_{13}^{k l}}{\partial \mathbf{x}}=\left[\left(\mathbf{e}^{k l}\right)^{T}, 0,0, \mathbf{0}_{1 \times N_{s a t}-1}, \mathbf{0}_{1 \times N_{s a t}-1}\right]
$$

$$
\begin{gathered}
\frac{\partial P_{23}^{k l}}{\partial \mathbf{x}}=\left[\left(\mathbf{e}^{k l}\right)^{T}, h_{\theta}, h_{\psi}, \mathbf{0}_{1 \times N_{s a t}-1}, \mathbf{0}_{1 \times N_{s a t}-1}\right] \\
\frac{\partial \phi_{13}^{k l}}{\partial \mathbf{x}}=\left[\left(\mathbf{e}^{k l}\right)^{T}, 0,0, \lambda_{\boldsymbol{k}}, \mathbf{0}_{1 \times N_{s a t}-1}\right] \\
\frac{\partial \phi_{23}^{k l}}{\partial \mathbf{x}}=\left[\left(\mathbf{e}^{k l}\right)^{T}, h_{\theta}, h_{\psi}, \mathbf{0}_{1 \times N_{s a t}-1}, \lambda_{k}\right]
\end{gathered}
$$

where $\lambda_{\boldsymbol{k}}$ is a vector of $N_{\text {sat }}-1$ zero, except for one value which is equal to $\lambda_{G P S}$ in the k-th position.

$$
\text { with } \begin{aligned}
h_{\theta} & =\left(\mathbf{e}^{k l}\right)^{T} *(-l) *\left[\begin{array}{c}
-\sin \theta \sin \varphi \\
-\sin \theta \cos \varphi \\
\cos \theta
\end{array}\right] \text { and } \\
h_{\psi} & =\left(\mathbf{e}^{k l}\right)^{T} *(-l) *\left[\begin{array}{c}
\cos \theta \cos \varphi \\
-\cos \theta \sin \varphi \\
0
\end{array}\right] \text { respectively. }
\end{aligned}
$$

Thus, the corresponding Matrix $\mathbf{H}$ can be defined as follows: 

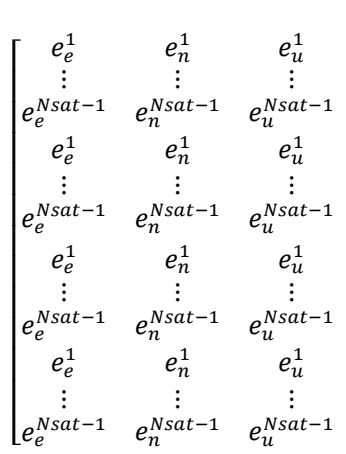

$\begin{array}{ccc} & \mathbf{H}= \\ 0 & 0 & 0 \\ \vdots & \vdots & \vdots \\ 0 & 0 & 0 \\ h_{\theta} & h_{\psi} & 0 \\ \vdots & \vdots & \vdots \\ h_{\theta} & h_{\psi} & 0 \\ 0 & 0 & \lambda^{G P S} \\ \vdots & \vdots & 0 \\ 0 & 0 & 0 \\ h_{\theta} & h_{\psi} & 0 \\ \vdots & \vdots & 0 \\ h_{\theta} & h_{\psi} & 0\end{array}$

$\begin{array}{rr} & \\ \ddots & 0 \\ \ddots & \vdots \\ \ldots & 0 \\ \ldots & 0 \\ \ddots & \vdots \\ \ldots & 0 \\ \ldots & 0 \\ \ddots & 0 \\ \ldots & \lambda^{G P} \\ \ldots & 0 \\ \ddots & 0 \\ \ldots & 0\end{array}$

0
$\vdots$
0
0
$\vdots$
0
0
0
0
$\lambda^{G P S}$
$\vdots$
0

$\begin{array}{cc}\cdots & 0 \\ \ddots & \vdots \\ \cdots & 0 \\ \cdots & 0 \\ \ddots & \vdots \\ \cdots & 0 \\ \cdots & 0 \\ \ddots & 0 \\ \cdots & 0 \\ 0 & 0 \\ \ddots & \vdots \\ 0 & \lambda^{G P S}\end{array}$

Note that the values of $\mathbf{H}$ are computed around the predicted position $\hat{\mathbf{x}}_{n}^{-}$

The update of the state vector is performed as:

$$
\hat{\mathbf{x}}_{n}^{+}=\hat{\mathbf{x}}_{n}^{-}+\mathbf{K}_{n}\left(\mathbf{z}_{n}-\mathbf{h}_{n}\left(\hat{\mathbf{x}}_{n}^{-}\right)\right)
$$

where $\mathbf{K}_{n}$ stands for the Kalman gain matrix and the term $\mathbf{z}_{n}-\mathbf{h}_{n}\left(\hat{\mathbf{x}}_{n}^{-}\right)$is defined as measurement innovation. This discrepancy between what the sensors are telling and what the linear model is pointing out is weighted by the Kalman gain matrix $\mathbf{K}_{n}$ and then finally added to the just calculated predicted state vector $\hat{\mathbf{x}}_{n}^{-}$coming from the last prediction phase.

The a-posteriori state covariance matrix is updated as follows:

$$
\mathbf{P}_{n}^{+}=\left(\mathbf{I}-\mathbf{K}_{n} \mathbf{H}_{n}\right) \mathbf{P}_{n}^{-}
$$

with $\mathbf{I}$ being the identity matrix, and $\mathbf{K}_{n}$ the Kalman gain matrix, which is defined as:

$$
\mathbf{K}_{n}=\mathbf{P}_{n}^{-} \mathbf{H}_{n}^{T}\left(\mathbf{H}_{n} \mathbf{P}_{n}^{-} \mathbf{H}_{n}^{T}+\mathbf{R}_{n}\right)^{-1}
$$

The updated state vector $\hat{\mathbf{x}}_{n}^{+}$contains now optimal state estimates, considering both the new information provided by the measurements and the prediction of the linear model. This procedure should be iterated as long as new measurement data is available.

\section{RTK PROCESSING}

In the previous process, we obtain a float estimation of the double-difference integer ambiguity. The accuracy of the position state estimate is further improved by fixing the DD ambiguities to integer number by using the well-known LAMBDA [11], [12] algorithm. This algorithm has shown its superiority in terms of both performance and processing time when compared to other algorithms in [13].

One selects the integer candidates based on the sum of squared errors to get a fixed solution. The candidate with the lowest error norm is chosen once the ratio of the Maximum A Posteriori error norm between the second-best candidate and the best candidate is bigger than a threshold. It is a pre-defined threshold or the critical value that the squared norm of ambiguity residuals of the best and second-best candidates should overpass to validate the integer estimation. In our paper, an empirical fixed value 3 is taken as in [14].

Once the IAR process is declared successful, a new position is computed using the DD carrier phase measurements corrected by the validated DD integer ambiguities. This final position is the fixed solution.

\section{CYCLE SLIP DETECTION AND REPAIR}

A cycle slip is a discontinuity of the measured carrier phase resulting from a temporary loss of lock in the carrier tracking loop of a GNSS receiver, the occurrence of a cycle slip describes the fact that the value of a carrier phase ambiguity term does not 31) hold constant between two consecutive epochs. According to [15], the 3 main causes of CS are signal obstruction, low C/N0, failure in the receiver software. A fourth cause pointed out by [16] is the receiver dynamic which may cause a phase error exceeding the PLL discrimination linear domain. Although usually relatively rare in static conditions, cycle slips occurrence can be very frequent for low-cost moving receivers. It is then very important to detect them and if it's possible to repair them in order to estimate ambiguities as constants.

When a cycle slip occurs, the corresponding ambiguity state in the KF shall be re-initialized, which will lead to the degradation of the estimated position accuracy. Preferably, the value of the CS should be estimated and added to the ambiguity state to maintain the high accuracy of fixed estimation. The aim of a CS-DR scheme is thus to detect the occurrence of CS and to enable the continuous use of constant carrier phase ambiguity when no CS is detected (either to be able to fix it or to be able to use its accurately-estimated value).

The entire cycle-slip processing is in theory composed of four sequential stages [17]: (1) cycle-slip detection to check for the occurrence of cycle-slips; (2) cycle-slip determination to quantify the sizes of cycle-slips; (3) cycle-slip validation to verify the determined sizes of cycle-slips; (4) cycle-slip removal to remove the cycle-slips out of the associated phase measurements. The steps (2) - (4) are also referred to as cycleslip repair or cycle-slip correction. Fig. 2 shows a typical CSDR process.

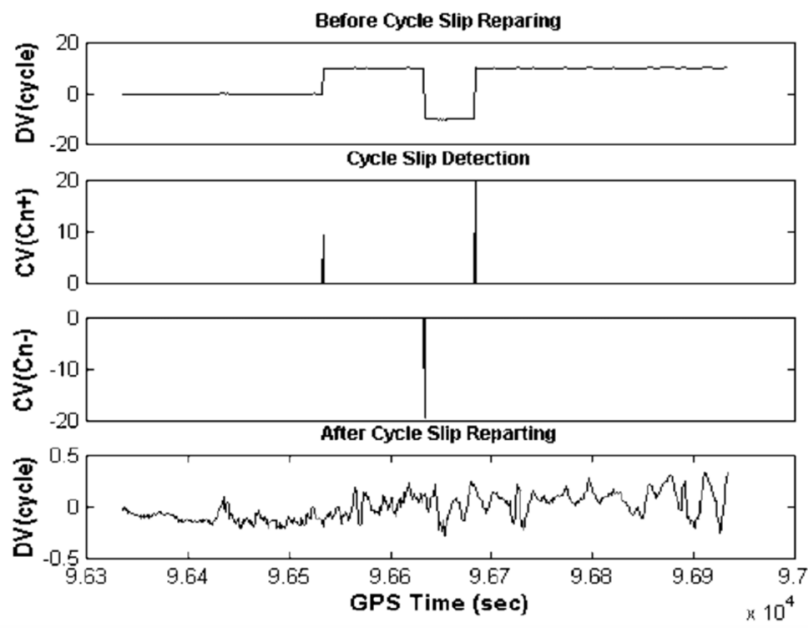

Fig. 2. Typical Cycle Slip Detection and Repair Process [18]

The proposed KF scheme assumes that the carrier phase measurements have a constant carrier phase ambiguity. However, it is well known that this does not necessarily hold for very long especially in an urban environment where frequent CSs occur. But assuming that a CS occurs at each epoch is detrimental to the PVT algorithm accuracy performance since it implies a constant re-estimation of the float ambiguity states 
without benefiting from their potential continuity. It is, however, the least risky. Consequently, it might be important to closely monitor the occurrence of CS continuously to follow the continuous-phase ambiguity model with confidence.

Cycle slip detection and repair are crucial to maintain continuity of carrier phase observations and to benefit the precise GPS carrier phase observations for high-precision GPS positioning. There are currently many methods to detect and repair the cycle slips. However, it is still a challenging issue, in particular in the case of the single-frequency measurements

In this paper, two types of cycle slip detection processes are studied. The Standard Phase-Code Comparison method and the Differential Phases of Time Cycle Slip Resolution Method.

\section{A. Standard Phase-Code Comparison method}

The Standard Phase-Code Comparison method is based on the observation of code-minus-carrier (CMC) combinations, The cycle slips of the phase observable can be detected by using the following formula [19]:

Where

$$
\Delta_{t} P=\lambda \Delta_{t} \Phi-\lambda \Delta_{t} N+\varepsilon
$$

- $\Delta_{t}$ is the time difference operator, used to differencing the observation equations between two adjacent epochs

- $\quad P$ refers to the code range measurement

- $\Phi$ refers to the carrier phase measurement

- $\quad N$ refers to the ambiguity

- $\lambda$ refers to the wavelength

- $\varepsilon$ refers to residual

Form (35), we can deduce that:

$$
\Delta_{t} N=\frac{\lambda \Delta_{t} \Phi-\Delta_{t} P}{\lambda}+\varepsilon
$$

This operation removes the geometry term and the other frequency-independent (or non-dispersive) terms, whereas it will enlarge the ionospheric error and introduce large thermal noise and potential multipath error from code data. The remaining errors are summarized by the term $\varepsilon$.

In the case of no cycle slips, the time difference of the ambiguity is zero, i.e. $\Delta_{t} N=0$. In the case of a cycle slip, $\Delta_{t} N$ $\neq 0$ and we can have the following formula:

$$
\left|\frac{\lambda \Delta_{t} \Phi-\Delta_{t} P}{\lambda}\right|>\varepsilon \text { if } \Delta_{t} N \neq 0
$$

The residual between the prediction and the observation can then be used as a detector metric, to be compared to detection threshold $\varepsilon$. The a priori value of $\varepsilon$ is determined according to the quality of code data and the potential multipath error and the desired false alarm probability. A larger value will lower the sensitivity, whereas tuning it down to a small number might cause a wrong detection. This approach facilitates only a rough detection of cycle-slips. It is not suitable for small cycle slips due to large measurement noise and multipath in code pseudorange. The minimal detectable size can be estimated by rounding $\varepsilon / \lambda_{j}$.

\section{B. Differential Phases of Time Cycle Slip Resolution Method}

The second cycle slip detection process [19] is based on the observation of the differential phases between two adjacent epochs, which should exhibit the actual ambiguity, plus some clock errors and remaining noise term as shown in (38):

$$
\lambda \Delta_{t} \Phi=\Delta_{t} \rho-\Delta_{t}\left(\delta t_{\mathrm{r}}-\delta t_{k}\right) c+\lambda \Delta_{t} N+\varepsilon_{\mathrm{p}}
$$

Where

- $\quad \rho$ refers to the geometric distance between the satellite and the receiver antennas

- $\delta t_{\mathrm{r}}, \delta t_{k}$ refer to the clock errors of the receiver and satellite respectively

- $\quad c$ is the speed of light

- $\varepsilon_{\mathrm{p}}$ refers to remaining errors of the phase measurement

Except for the ambiguity term, all other terms on the right side are of low variation. Any cycle slips will lead to a sudden jump of the time difference of the phases. Based on the past observation of differential phases measurement, a prediction of the current differenced data can be obtained by polynomial extrapolation or interpolation [10]. The residual between the prediction and the observation can then be used as a detector metric, to be compared to the detection threshold to decide if there are any cycle slips. Note that this detector works independently for each phase measurement.

\section{Cycle slip validation, size determination, and repair}

The last process of cycle slip detection is to set a proper threshold for the detection value. In cycle slip detection, the detection threshold is the magnitude of the detection metric that must be exceeded to consider the occurrence of a cycle slip. An optimal threshold should always be sensitive to small cycle slips. In our model, the threshold is determined by the metric noise and the significance level:

$$
\text { Threshold }=\sqrt{2} * \alpha * \sigma_{m}
$$

Where

- $\quad \alpha$ refers to the significance level related coefficient

$$
\text { ( e. } g: \alpha=2 \text {, significance level is } 0.05 \text { ) }
$$

- $\sigma_{m}$ refers to the standard deviation of the considered metric, which corresponds to $\sigma_{\text {code }}$ for the Standard Phase-Code Comparison method and $\sigma_{\text {phase }}$ for the Differential Phases of Time Cycle Slip Resolution Method

After the cycle slips detection process, a cycle-slip validation and size determination process is needed to verify the determined sizes of cycle-slip. Cycle slips can be repaired using integer vector estimation similarly to ambiguity resolution in the position domain.

After detecting all the possible cycle slips at a given epoch, one may obtain the estimates of the float value cycle slips $x_{j}(j$ ranges from 1 to number of detected cycle slips) together with their covariance matrix. Then, an integer estimation technique is employed to determine the integer cycle slip vector from the 
float cycle slips $x_{j}$ and their covariance matrix. In our case, this is achieved by means of the least squares ambiguity decorrelation adjustment (LAMBDA) method [11], [12]. An advantage of the LAMBDA method is that it comprises both an estimation step to determine the integer value of the cycle slips, and a validation step, to determine if the confidence level associated with the estimate is sufficient for the user. If LAMBDA gives an integer cycle slip value equals to 0 at one specific epoch, it means that the cycle slip has not taken place at that epoch which means we have a false detection. Another case is that if the cycle slip is detected by the metric, but that LAMBDA does not provide an integer solution with enough confidence, we re-initialize the state associated with the ambiguity of the satellite in the state vector to ensure a better RTK performance.

Once the sizes of cycle slips are determined, we conduct the cycle-slip removal (repair) step to remove the cycle slips out of the associated phase measurements.

\section{Centralized cycle slips detection using an array of receiver}

In our model, the cycle slips are simulated as correlated in both the receivers, with a correlation of $100 \%$ for the occurrence of simultaneous cycle slips on the same satellite. The reason that we chose this assumption is that here we consider that the cycle slip is due to an event external to the receiver, which is mostly the case in reality, such as an obstruction or severe multipath environment. In this case, there could be a strong correlation between the occurrence of cycle slips in the 2 receivers who are very close to each other. This means that if the cycle slip occurs on one receiver, then there is a great chance $(100 \%$ in the simulated scenario) that it occurs for the other receiver, even though the amplitudes of the simultaneous cycle slips are not necessarily correlated.

So, in this article, a centralized cycle slips detection methodology is used. Which means that there are 3 different possible situations:

1) The algorithm detects that there is a cycle slip in both the receivers at a specific epoch: cycle slip validation and determination process are needed for both the receivers in this epoch.

2) The algorithm detects that there is a cycle slip in one receiver at a specific epoch, while no cycle slip in another receiver: we consider there is also a cycle slip occurring in the other receiver at the same epoch. We then conduct the cycle slip validation, size determination process for both the receivers separately to validate and repair their own cycle slip.

3) The algorithm detects no cycle slip in both the receivers at a specific epoch: no cycle slip validation process needed.

In this situation, the use of observations from the two receivers of the array will double the chance to detect the possible small cycle slips, which can increase the probability of successful detection, so it is expected that an array of receivers can improve the performance of the cycle slip detector.

\section{SIMULATION OF CS-DR PROCESS AND RTK PERFORMANCES USING AN ARRAY OF RECEIVERS}

In this section, the proposed precise position and attitude determination algorithm is verified with simulated measurements. The reason why we use the simulated measurements is that we want to see the advantage of using an array of receivers with known geometry in CS-DR process in controlled scenarios with varying the occurrence rate of the $\mathrm{CS}$, the sizes of the CSs and the noise measurement when compared to the traditional single receiver system. To validate the interest of the use of an array of receivers, several scenarios are conducted, and conclusions are drawn from the comparison between single receiver RTK and multi-receiver RTK in terms of percentage of epochs with integer cycle slip correctly resolved, fixing rate and position accuracy.

The performance criteria shown in the presented tables are obtained by computing the mean, standard deviation, and $95 \%$ bound of indicators, based on the running of 100 Monte-Carlo simulations, where the noise and initial states are drawn randomly.

\section{A. System Configurations}

To get enough change of the attitude of the vehicle during the simulation so as to observe the algorithm performance for different heading and pitch, the vehicle is assumed to make a uniform circular motion around the fixed reference receiver 3 with a constant radius $(\mathrm{R}=100 \mathrm{~m})$ in all the scenarios. The array baseline length between two rover receivers is set to $l=2 \mathrm{~m}$.

The relative horizontal positions of the dual-receiver system and the single receiver system in time are shown in Fig.3 and Fig.4, respectively.

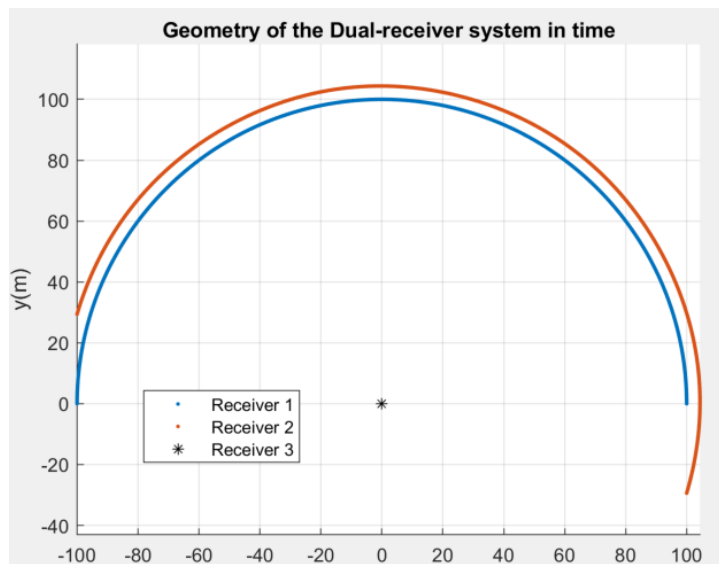

Fig. 3. The geometry of the Dual-receiver system in time

In order to make the pitch angle and the relative altitude between receiver 1 and 2 observable, the ground is considered to be undulated thus we also add a small altitude and pitch variation as shown in Fig.5. The speed of the vehicle is $10 \mathrm{~m} / \mathrm{s}$, the duration of the simulated trajectory is $1000 \mathrm{~s}$ and the sampling rate is $1 \mathrm{~Hz}$.

For readability purposes, the length between the 2 rover receiver antennas is set to $l=10 \mathrm{~m}$ in Fig. 3 and 5, while it is 2 $\mathrm{m}$ in the simulation. 


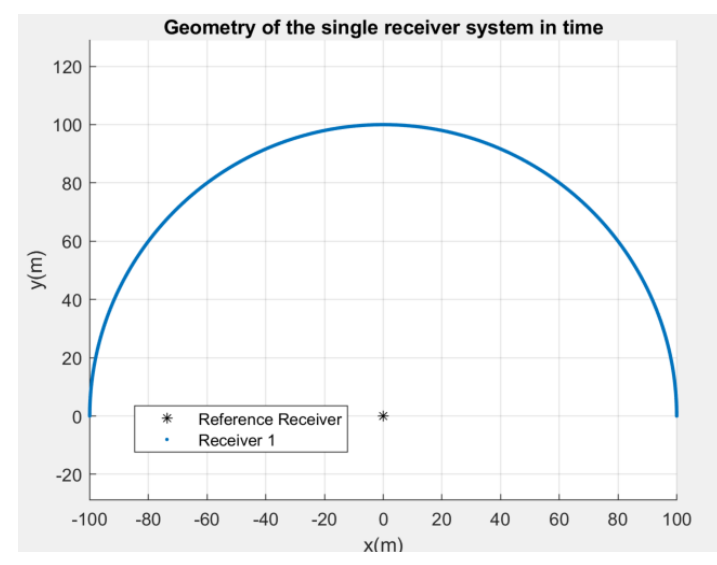

Fig. 4. The geometry of the Single-receiver system in time

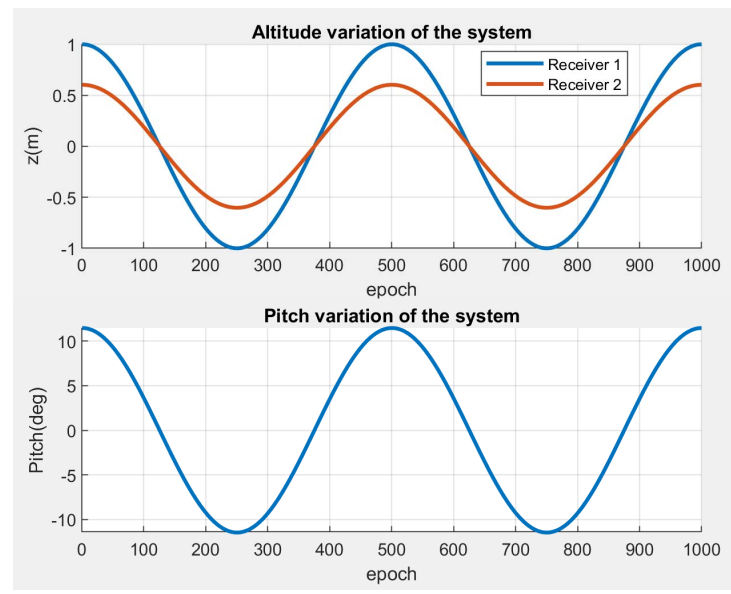

Fig. 5. Altitude and Pitch angle variation of the Dual-Receiver system

In our simulator, the satellites are simulated with a fixed position as shown in Fig.6. We consider 7 satellites with fairly good geometry, i.e. well-spaced in azimuth and elevation. This would correspond to a typical case of a single constellation in an open sky environment.

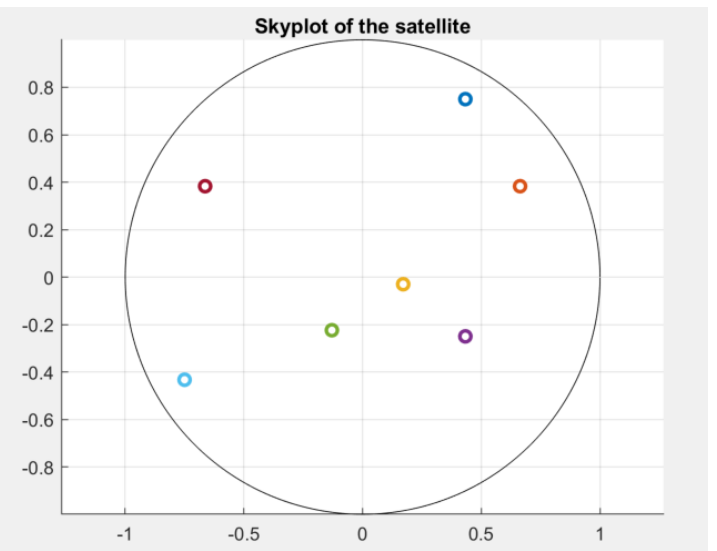

Fig. 6. Considered satellite geometry

Finally, the initial DD carrier ambiguities are taken as fixed values separated by 10 cycles. This means that the DD ambiguities go from 10 to 120 .
During the observation period, $m$ simulated cycle slips were manually added to the simulated data to test the cycle slip detection and repair process. For each satellite, the $m$ cycle slips were consecutively added to its carrier phase measurements from their random given start epoch. The sizes of the cycle slips are predefined fixed values which depend on the ongoing scene.

Fig. 7 gives an example of the distribution of the simulated cycle slip for each satellite during the simulation. The added cycle slips are depicted in the red cross as shown in the figure.

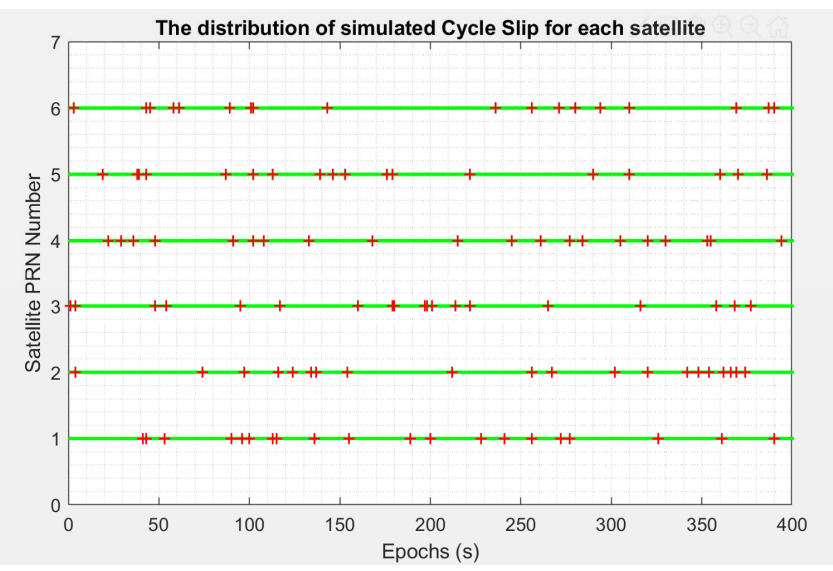

Fig. 7. The distribution of simulated Cycle Slip for each satellite

\section{B. Typical results of the RTK state estimations using array receiver system}

Typical results of the state estimations including the estimation of the integer ambiguity resolution (last row) by using LAMBDA are given in Fig.8. The top 3 rows show the estimation of the East, North and Up position coordinate respectively whereas the 4 th and 5 th rows show the estimation of the pitch and heading angles of the vehicle.

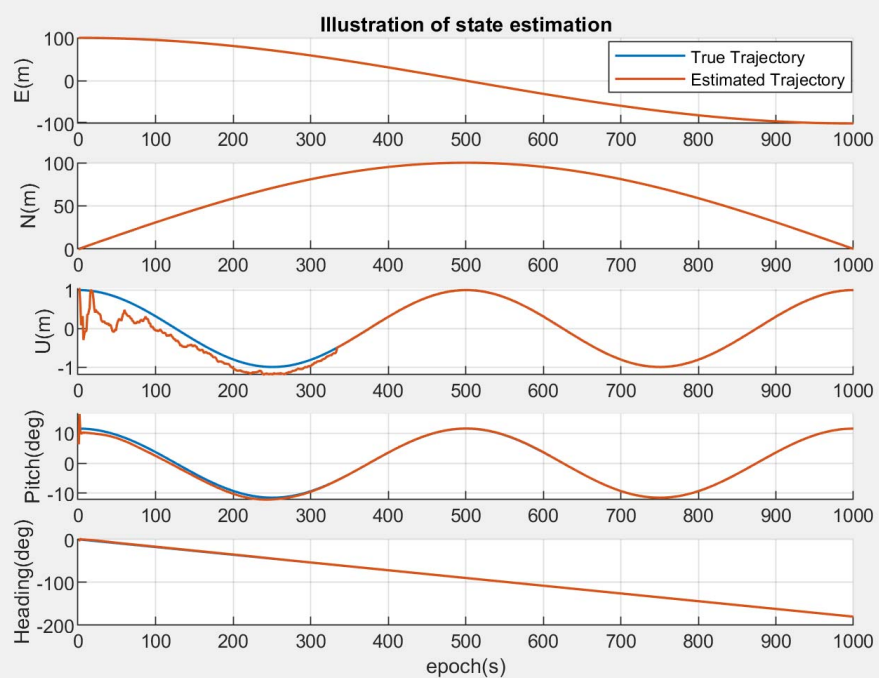

Fig. 8. Illustration of state estimation

Fig.9 gives the error comparison between the float and fixed solution. We can see from the result that some convergence 
period is present in all states. Once they converge, the error between the estimated result and the true value is extremely small.

In Fig.9, the fixed solution is defined by two parts, for every epoch, if the IAR process is declared successful, a new position is computed using the DD carrier phase measurements corrected by the validated DD integer ambiguities. This position is then used as the fixed solution. If the IAR process is not declared successful, we keep the float solution.

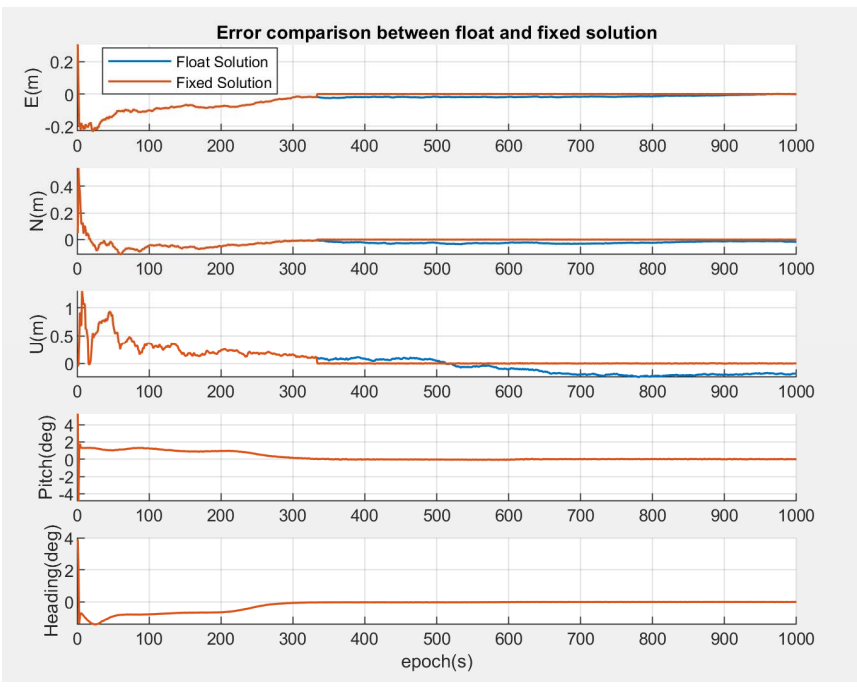

Fig. 9. Illustration of float vs fixed solution

From the figure, we can see that the system fixes the IAR in the 322 nd epoch and once the IAR is fixed, the positioning results become much more accurate compared to the float solution. However, in terms of the heading and the pitch, the precision is not much improved in the fixed solution because its precision is already good enough in the float solution.

\section{Typical results of the Cycle-Slips Detection using array receiver system}

According to our previous work [10], our proposed dual receiver array system provides always better performance than the single receiver RTK solution when under the same condition (e.g. Noise amplitude $\sigma_{\text {code }}=\mathbf{1} \mathrm{m}, \boldsymbol{\sigma}_{\text {phase }}=\mathbf{1} \mathbf{~ m m}$, Visible Satellite Number $\boldsymbol{N}_{\text {sat }}=\mathbf{7}$, length of the vehicle antenna baseline $\boldsymbol{l}=\mathbf{2} \mathrm{m}$, Cycle Slip free) as shown in Table I

TABLE I. SINGLE/DUAL RECEIVER RTK PERFORMANCE COMPARISON IN A CS-FREE SCENARIO

\begin{tabular}{|c|c|c|c|c|c|}
\hline System & Performance indicator & Unit & Mean & $\begin{array}{c}\text { Standard } \\
\text { deviation }\end{array}$ & $\begin{array}{c}95 \% \\
\text { bound }\end{array}$ \\
\hline \multirow{2}{*}{$\begin{array}{c}\text { Single } \\
\text { receiver }\end{array}$} & 3D position error & $\mathrm{m}$ & 0.1043 & 0.1591 & 0.0132 \\
\cline { 2 - 6 } & Absolute heading error & $\mathrm{deg}$ & $\mathrm{N} / \mathrm{A}$ & $\mathrm{N} / \mathrm{A}$ & $\mathrm{N} / \mathrm{A}$ \\
\cline { 2 - 6 } & Absolute pitch error & $\mathrm{deg}$ & $\mathrm{N} / \mathrm{A}$ & $\mathrm{N} / \mathrm{A}$ & $\mathrm{N} / \mathrm{A}$ \\
\cline { 2 - 6 } & Fixed rate & $\%$ & 93.35 & 12.08 & 66.38 \\
\hline \multirow{2}{*}{$\begin{array}{c}\text { Dual } \\
\text { receiver }\end{array}$} & 3D position error & $\mathrm{m}$ & 0.0885 & 0.1940 & 0.0019 \\
\cline { 2 - 6 } & Absolute heading error & $\mathrm{deg}$ & 1.8666 & 2.8054 & 0.0071 \\
\cline { 2 - 6 } & Absolute pitch error & $\mathrm{deg}$ & 1.0524 & 1.2755 & 0.0455 \\
\cline { 2 - 6 } & Fixed rate & $\%$ & 96.69 & 10.37 & 77.14 \\
\hline
\end{tabular}

To conduct the CS detection, both methods proposed in section V. need the raw GNSS measurement. Fig.10 shows our simulated code and carrier phase measurement on the L1 frequency with noise $\sigma_{\text {phase }}=10 \mathrm{~mm}, \sigma_{\text {code }}=1 \mathrm{~m}$ and a sampling interval of $1 \mathrm{~s}$ for PRN 03 based on a real almanac on February 04, 2020. Both of carrier phase and pseudorange measurements are plotted versus time. The Carrier phase is multiplied with its wavelength to be presented in units of meters.

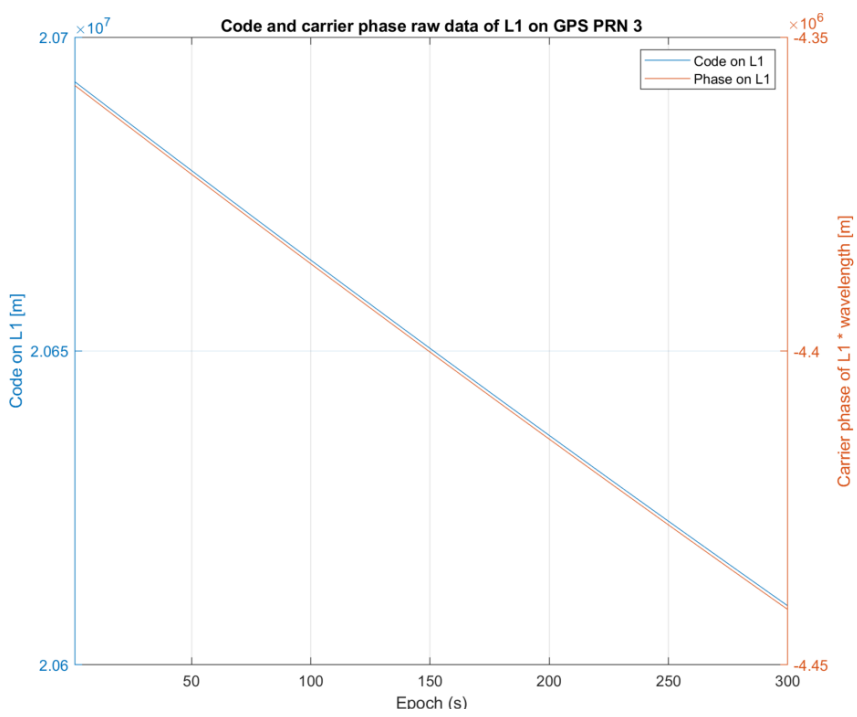

Fig. 10. Simulation L1 code and carrier phase data

Ten cycle slips with the size of 1,2 , or 4 cycles have been added to the original L1 carrier phase observation of Fig. 10 at the epoch instant of $100,120, \ldots, 280$, respectively, as shown in the Fig. 11 .

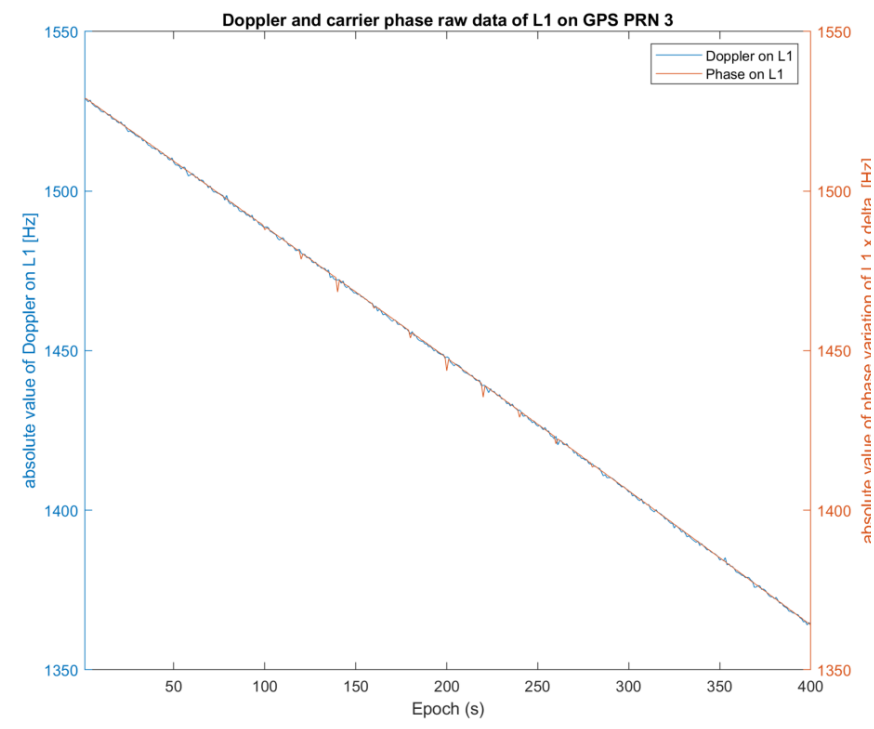

Fig. 11. Simulation L1 carrier phase and doppler data

The two proposed methods are now applied to this data set.

Fig.12 shows the result of the Standard Phase-Code Comparison method, in the figure, the black point stands for the difference between the between-epoch phase variation and code variation, the green line is the threshold which is dependent on 
the code quality identified by the predefined $\sigma_{\text {phase }}$ and $\sigma_{\text {code }}$, with significance level related coefficient $\alpha=2$ to make the significance level equals to 0.05 .

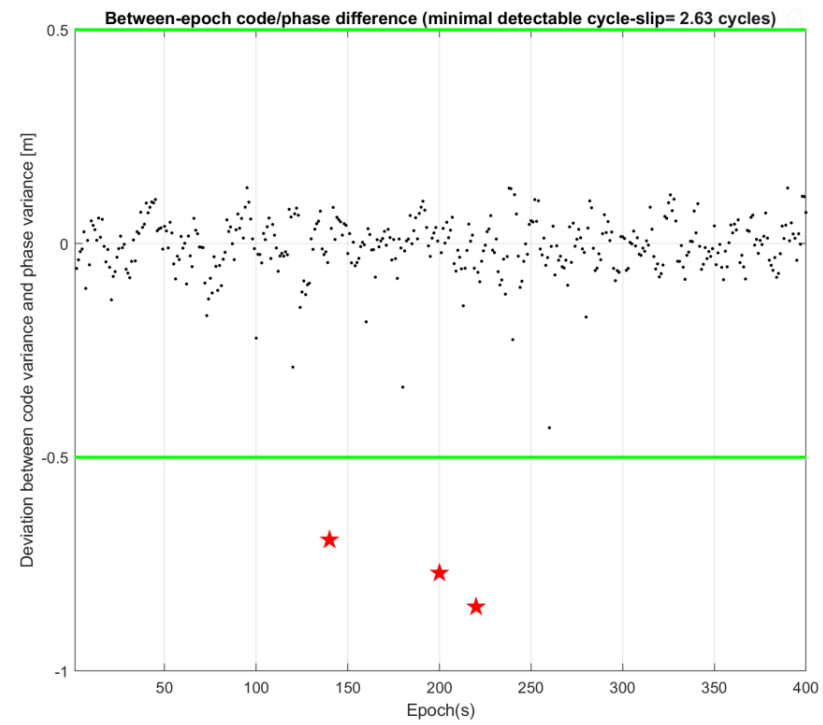

Fig. 12. The result of the Standard Phase-Code Comparison method

Consider fig.12, one can see that there are several outlier points that correspond to the added cycle-slips. However, the difference is not big enough to pass the threshold thus these cycle slips are not detected successfully. Only the big cycle slips whose size is 4 are detected. In our case with the predefined standard deviation value of the phase and code noise, the minimal detectable cycle slip is 2.86 cycles which is too large to enable the detection of the small cycle-slips.

Unlike the first method, the Differential Phases of Time Cycle Slip Resolution Method shows its superiority in detecting the small cycle slips.

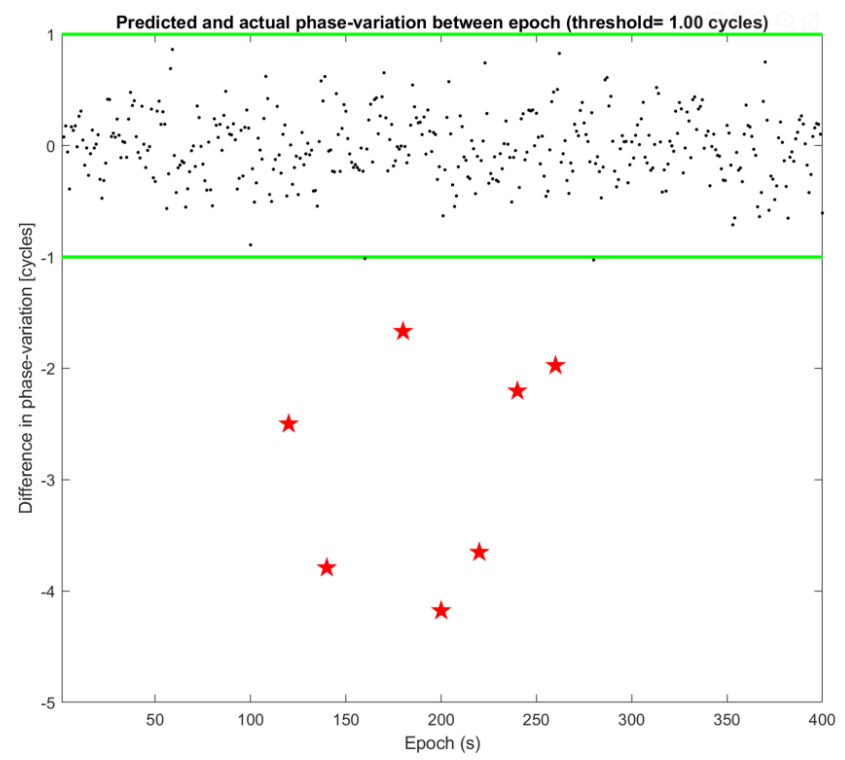

Fig. 13. The result of the Differential Phases of Time Cycle Slip Detection Method
As can be seen in Fig.13, a large jump indicates a cycle slip, as shown by the red star. More added cycle slips are detected timely and accurately, only some of the smaller cycle slips $($ size $=1)$ are not detected. Still, this method is much better than the first method, because the noise level of the code measurements is much higher than that of the phase, the Standard Phase-Code Comparison method can be used only for the detection of large cycle slips.

Thus, in the following part of this article, we will only present the CS detection result by using the Differential Phases of Time Cycle Slip Resolution Method.

After detecting the occurrence of cycle slips, we apply the LAMBDA method to determine the integer cycle slip vector from the suspected cycle slips epochs then conduct the cycleslip repair step to remove the cycle slips out of the associated phase measurements.

In the following sections, one will analyze the CS-DR performance and the related RTK result of the dual receiver system and the single receiver system by conducting 3 different scenarios:

1) The Noise measurement effect Analyses

2) Impact of the sizes of the added Cycle slips

3) Impact of the occurrence rate of the added Cycle slips

\section{Noise measurement effect Analyses}

The level of the noise measurement has a crucial influence on the estimation of the cycle slip detection metric which will decide whether there is a cycle slip or not, thus it is a significant parameter that needs to be analyzed.

In the first scenario, the size and the occurrence rate of the added Cycle slip are fixed, while varying the level of the noise measurement $\sigma_{\text {phase }}$, we observe the robustness against the noise of our proposed multi-receivers RTK system.

The analysis of Table II shows that the use of the receiver array improves the correctly CS-DR rate for all the bad noise level situations, thus demonstrating the interest of such an approach.

TABLE II. CS-DR COMParison With CyCle SliP (Size = 2), Mean No. CS $=\mathbf{2 0}$

\begin{tabular}{|c|c|c|c|}
\hline System & $\begin{array}{c}\text { No. of } \\
\text { simulated } \\
\text { CS }\end{array}$ & $\begin{array}{c}\text { Mean No. of correctly } \\
\text { detected and repaired } \\
\text { cycle slips }\end{array}$ & $\begin{array}{c}\text { Mean } \\
\text { Corrected } \\
\text { Rate }\end{array}$ \\
\hline $\begin{array}{c}\text { Single receiver } \\
\sigma_{\text {phase }}=5 \mathrm{~mm}\end{array}$ & 20 & 20 & $100 \%$ \\
\hline $\begin{array}{c}\text { Dual receiver } \\
\sigma_{\text {phase }}=\mathbf{5} \mathrm{mm}\end{array}$ & 20 & 20 & $100 \%$ \\
\hline $\begin{array}{c}\text { Single receiver } \\
\sigma_{\text {phase }}=\mathbf{2 0} \mathrm{mm}\end{array}$ & 20 & 17.8 & $89 \%$ \\
\hline $\begin{array}{c}\text { Dual receiver } \\
\sigma_{\text {phase }}=20 \mathrm{~mm}\end{array}$ & 20 & 18.5 & $92.5 \%$ \\
\hline $\begin{array}{c}\text { Single receiver } \\
\sigma_{\text {phase }}=\mathbf{5 0} \mathrm{mm}\end{array}$ & 20 & 9.5 & $42.5 \%$ \\
\hline $\begin{array}{c}\text { Dual receiver } \\
\sigma_{\text {phase }}=\mathbf{5 0} \mathrm{mm}\end{array}$ & 20 & 11.8 & $55.4 \%$ \\
\hline
\end{tabular}


In terms of the RTK related parameters, one can see from Table III that the dual receiver array system provides better performance than the single receiver RTK solution, thus demonstrating the interest of such an approach.

TABLE III. RTK PERFORMANCE COMPARISON FOR THE DIFFERENT SYSTEM WITH CYCLE SLIP (SIZE = 2), MEAN No.CS =20

\begin{tabular}{|c|c|c|c|c|c|}
\hline System & Terms & Unit & Mean & Std & $\begin{array}{l}95 \% \\
\text { bound }\end{array}$ \\
\hline \multirow{2}{*}{$\begin{array}{l}\text { Single receiver } \\
\boldsymbol{\sigma}_{\text {phase }}=\mathbf{5} \mathrm{mm}\end{array}$} & $3 D$ error & $\mathrm{m}$ & 0.1725 & 0.2591 & 0.0272 \\
\hline & Fixed rate & $\%$ & 92.35 & 16.08 & 51.13 \\
\hline \multirow{2}{*}{$\begin{array}{l}\text { Dual receiver } \\
\sigma_{\text {phase }}=\mathbf{5} \mathrm{mm}\end{array}$} & 3D error & $\mathrm{m}$ & 0.1185 & 0.1940 & 0.0018 \\
\hline & Fixed rate & $\%$ & 96.69 & 12.37 & 67.23 \\
\hline \multirow{2}{*}{$\begin{array}{l}\text { Single receiver } \\
\boldsymbol{\sigma}_{\text {phase }}=\mathbf{2 0} \mathrm{mm}\end{array}$} & 3D error & $\mathrm{m}$ & 0.2815 & 0.4649 & 0.0716 \\
\hline & Fixed rate & $\%$ & 66.25 & 18.04 & 28.82 \\
\hline \multirow{2}{*}{$\begin{array}{l}\text { Dual receiver } \\
\sigma_{\text {phase }}=\mathbf{2 0} \mathrm{mm}\end{array}$} & $3 \mathrm{D}$ error & $\mathrm{m}$ & 0.2247 & 0.2876 & 0.0322 \\
\hline & Fixed rate & $\%$ & 69.40 & 13.60 & 32.56 \\
\hline \multirow{2}{*}{$\begin{array}{l}\text { Single receiver } \\
\boldsymbol{\sigma}_{\text {phase }}=\mathbf{5 0} \mathrm{mm}\end{array}$} & 3D error & $\mathrm{m}$ & 0.3846 & 0.4824 & 0.1512 \\
\hline & Fixed rate & $\%$ & 19.44 & 4.47 & 11.94 \\
\hline \multirow{2}{*}{$\begin{array}{l}\text { Dual receiver } \\
\boldsymbol{\sigma}_{\text {phase }}=\mathbf{5 0} \mathrm{mm}\end{array}$} & 3D error & $\mathrm{m}$ & 0.2768 & 0.3584 & 0.0577 \\
\hline & Fixed rate & $\%$ & 24.98 & 6.58 & 12.39 \\
\hline
\end{tabular}

\section{E. Impact of the size of the added Cycle slips}

Obviously, the smaller the size of the cycle-slip, the more difficult to detect and repair it. In this second scenario, we analyze the impact of the size of the added Cycle slips on the single receiver system and our proposed multi-receivers RTK system.

The result in Table IV. indicates that when the size of the cycle-slip is big enough, both the systems can perfectly detect and repair the cycle-slips. While the multi-receiver system shows its advantage when dealing with the small size cycle-slip situation.

TABLE IV. CS-DR COMPARISON WITH $\boldsymbol{\sigma}_{\text {phase }}=\mathbf{1 0}$ MM, MEAN No.CS $=\mathbf{2 0}$

\begin{tabular}{|c|c|c|c|}
\hline System & $\begin{array}{c}\text { No. of } \\
\text { simulated } \\
\text { CS }\end{array}$ & $\begin{array}{c}\text { Mean No. of correctly } \\
\text { detected and repaired } \\
\text { cycle slips }\end{array}$ & $\begin{array}{c}\text { Mean } \\
\text { Corrected } \\
\text { Rate }\end{array}$ \\
\hline $\begin{array}{c}\text { Single receiver } \\
\text { (CS Size = 1) }\end{array}$ & 20 & 13.5 & $67.5 \%$ \\
\hline $\begin{array}{c}\text { Dual receiver } \\
\text { (CS Size = 1) }\end{array}$ & 20 & 13.8 & $69 \%$ \\
\hline $\begin{array}{c}\text { Single receiver } \\
\text { (CS Size = ) }\end{array}$ & 20 & 18.16 & $90.8 \%$ \\
\hline $\begin{array}{c}\text { Dual receiver } \\
\text { (CS Size = 2) }\end{array}$ & 20 & 18.83 & $94.1 \%$ \\
\hline $\begin{array}{c}\text { Single receiver } \\
\text { (CS Size = 4) }\end{array}$ & 20 & 20 & $100 \%$ \\
\hline $\begin{array}{c}\text { Dual receiver } \\
\text { (CS Size = 4) }\end{array}$ & 20 & 20 & $100 \%$ \\
\hline
\end{tabular}

In terms of the RTK related parameters, one can see from the table $\mathrm{V}$ that, when the size of the added cycle slip is too small, our CS-DR cannot successfully remove all the cycle slips, thus the fixing rate of our RTK system drops sharply has led to greatly increased inaccuracy in positioning.
It should be pointed out that the accuracy and fixing rate difference between the two systems are not large, however, the dual receiver array always improves slightly the fixing rate which confirms the interest of our approach.

TABLE V. RTK PERFORMANCE COMPARISON FOR THE DIFFERENT SYSTEM WITH $\sigma_{\text {phase }}=\mathbf{1 0}$ MM, MEAN NO.CS $=\mathbf{2 0}$

\begin{tabular}{|l|l|l|c|c|c|}
\hline \multicolumn{1}{|c|}{ System } & Terms & Unit & Mean & Std & $\begin{array}{l}\text { 95\% } \\
\text { bound }\end{array}$ \\
\hline \multirow{2}{*}{$\begin{array}{l}\text { Single receiver } \\
\text { (CS Size = 1) }\end{array}$} & 3D error & $\mathrm{m}$ & 0.6264 & 0.2324 & 0.4812 \\
\cline { 2 - 6 } & Fixed rate & $\%$ & 0.82 & 0.49 & 0.11 \\
\hline $\begin{array}{l}\text { Dual receiver } \\
\text { (CS Size = 1) }\end{array}$ & 3D error & $\mathrm{m}$ & 0.5432 & 0.3618 & 0.0758 \\
\cline { 2 - 6 } & Fixed rate & $\%$ & 1.86 & 1.20 & 0.18 \\
\hline \multirow{2}{*}{$\begin{array}{l}\text { Single receiver } \\
\text { (CS Size = 2) }\end{array}$} & 3D error & $\mathrm{m}$ & 0.5111 & 0.4649 & 0.3116 \\
\cline { 2 - 6 } & Fixed rate & $\%$ & 61.92 & 19.17 & 52.11 \\
\hline \multirow{2}{*}{$\begin{array}{l}\text { Dual receiver } \\
\text { (CS Size = 2) }\end{array}$} & 3D error & $\mathrm{m}$ & 0.4194 & 0.2276 & 0.0322 \\
\cline { 2 - 6 } & Fixed rate & $\%$ & 64.40 & 13.60 & 66.08 \\
\hline \multirow{2}{*}{$\begin{array}{l}\text { Single receiver } \\
\text { (CS Size = 4) }\end{array}$} & 3D error & $\mathrm{m}$ & 0.1725 & 0.2591 & 0.0272 \\
\cline { 2 - 6 } & Fixed rate & $\%$ & 89.35 & 15.18 & 51.13 \\
\hline \multirow{2}{*}{$\begin{array}{l}\text { Dual receiver } \\
\text { (CS Size = 4) }\end{array}$} & 3D error & $\mathrm{m}$ & 0.1323 & 0.1546 & 0.0019 \\
\cline { 2 - 6 } & Fixed rate & $\%$ & 91.69 & 14.37 & 54.23 \\
\hline
\end{tabular}

\section{F. Impact of the occurrence rate of the added Cycle slips}

The method that we utilize to detect the Cycle slips is based on the previous observation of the raw measurement, a frequent appearance of cycle-slip may make the detection process becomes more difficult.

So, in the third scenario, we want to see the performance when the two systems deal with the diverse occurrence rates of the added Cycle slip, this is realized by adding the different numbers of the cycle slip during the same 400s observation period.

TABLE VI. CS-DR COMPARISON WITH $\boldsymbol{\sigma}_{\text {phase }}=\mathbf{1 0} \mathrm{MM}, \mathbf{C Y C L E}$ $\operatorname{SLIP}(\operatorname{SIZE}=2)$

\begin{tabular}{|c|c|c|c|}
\hline System & $\begin{array}{c}\text { No. of } \\
\text { simulated } \\
\text { CS }\end{array}$ & $\begin{array}{c}\text { Mean No. of correctly } \\
\text { detected and repaired } \\
\text { cycle slips }\end{array}$ & $\begin{array}{c}\text { Mean } \\
\text { Corrected } \\
\text { Rate }\end{array}$ \\
\hline Single receiver & 20 & 18.16 & $90.8 \%$ \\
\hline Dual receiver & 20 & 18.83 & $94.1 \%$ \\
\hline Single receiver & 40 & 36.72 & $91.8 \%$ \\
\hline Dual receiver & 40 & 37.56 & $93.9 \%$ \\
\hline Single receiver & 80 & 73.12 & $91.4 \%$ \\
\hline Dual receiver & 80 & 75.44 & $94.3 \%$ \\
\hline
\end{tabular}

As follows from Table VI. shown above, the occurrence rate of the added cycle slips does not have much influence on the CSDR success rate. The main reason is due to our detector works independently for each phase measurement, so it is reasonable to have such a result. 


\section{CONCLUSION AND FUTURE WORK}

In this paper, we present a method includes an array of receivers with known geometry to enhance the performance of the CS-DR process for RTK positioning in different environments. Taking advantage of the attitude information and the known geometry of the array of receivers, we are able to improve some internal steps of precise position computation and the cycle slip detection and repair process.

From the simulations that have been carried out, it is possible to conclude that our multi-receiver RTK system is more robust to cycle slips with lower amplitude or a higher level of the noise measurement in the data, in terms of percentage of epochs with integer cycle slip correctly detected and repaired. In fact, by taking advantage of the diverse data observations from an array of receivers with known geometry, the prediction accuracy of the differential phase in time is ameliorated, thus the occurrence of CS can be detected more accurately and timely. Besides, consistent with the results of our previous work, we always get a better positioning accuracy under the same conditions when comparing with the single receiver system.

Based on this proof-of-concept, several future works are possible. Other types of CS-DR techniques could be tested, notably those who would benefit from the improved accuracy of the receiver array solution. The current simulator could be improved in order to consider more realistic conditions, such as elevation-dependent measurement noise. Arrays with more than 2 receivers could also be assessed, as well as the consideration of several receivers connected to the same antenna (zero vehicle antenna baseline configuration).

We also plan to investigate the interest of such an array for the mitigation of NLOS conditions permitted by the spatial diversity of the array.

Finally, the concept could be tested against real conditions by setting-up an experiment on a test vehicle. Notably, the observation and modeling of cycle slip occurrence on a receiver array would bring interesting inputs for this type of system.

\section{ACKNOWLEDGMENT}

This work has been supported by the China Scholarship Council (CSC). It is the Chinese Ministry of Education's nonprofit organization that provides support for international academic exchange with China.

\section{REFERENCES}

[1] M. Iafrancesco, "GPS/INS Tightly coupled position and attitude determination with low-cost sensors Master Thesis," p. 69.

[2] P. Henkel and C. Gunther, "Attitude determination with low-cost GPS/ INS," p. 9.

[3] P. Henkel, P. Berthold, and J. J. Kiam, "Calibration of magnetic field sensors with two mass-market GNSS receivers," in 2014 11th Workshop on Positioning, Navigation and Communication (WPNC), Dresden, Germany, 2014, pp. 1-5, doi: 10.1109/WPNC.2014.6843306.

[4] D. Medina, A. Heselbarth, R. Buscher, R. Ziebold, and J. Garcia, "On the Kalman filtering formulation for RTK joint positioning and attitude quaternion determination," in 2018 IEEE/ION Position, Location and Navigation Symposium (PLANS), Monterey, CA, 2018, pp. 597-604, doi: 10.1109/PLANS.2018.8373432.

[5] F. Aghili and A. Salerno, "Attitude determination and localization of mobile robots using two RTK GPSs and IMU," in 2009 IEEE/RSJ International Conference on Intelligent Robots and Systems, St. Louis, MO, USA, 2009, pp. 2045-2052, doi: 10.1109/IROS.2009.5354770.

[6] F. Aghili and A. Salerno, "3-D Localization of mobile robots and its observability analysis using a pair of RTK GPSs and an IMU," in 2010 IEEE/ASME International Conference on Advanced Intelligent Mechatronics, Montreal, QC, Canada, 2010, pp. 303-310, doi: 10.1109/AIM.2010.5695718.

[7] N. Nadarajah, A. Khodabandeh, K. Wang, M. Choudhury, and P. Teunissen, "Multi-GNSS PPP-RTK: From Large- to Small-Scale Networks," Sensors, vol. 18, no. 4, p. 1078, Apr. 2018, doi: $10.3390 / \mathrm{s} 18041078$.

[8] A. Khodabandeh and P. J. G. Teunissen, "Single-Epoch GNSS Array Integrity: An Analytical Study," in VIII Hotine-Marussi Symposium on Mathematical Geodesy, vol. 142, N. Sneeuw, P. Novák, M. Crespi, and F. Sansò, Eds. Cham: Springer International Publishing, 2015, pp. 263272.

[9] A. Khodabandeh and P. J. G. Teunissen, "Array-based satellite phase bias sensing: theory and GPS/BeiDou/QZSS results," Meas. Sci. Technol., vol. 25, no. 9, p. 095801, Sep. 2014, doi: 10.1088/09570233/25/9/095801.

[10] X. Hu, P. Thevenon, and C. Macabiau, "Improvement of RTK Performances Using an Array of Receivers with Known Geometry," presented at the 2020 International Technical Meeting of The Institute of Navigation, San Diego, California, Feb. 2020, pp. 440-453, doi: $10.33012 / 2020.17154$.

[11] P. J. G. Teunissen, "The least-squares ambiguity decorrelation adjustment: a method for fast GPS integer ambiguity estimation," $J$. Geod., vol. 70, no. 1-2, pp. 65-82, Nov. 1995, doi: 10.1007/BF00863419.

[12] P. Buist, "The Baseline Constrained LAMBDA Method for Single Epoch, Single Frequency Attitude Determination Applications," in 20th International Technical Meeting of The Satellite Division of the Institute of Navigation, Fort Worth, Texas, 2007.

[13] L. Baroni and H. K. Kuga, "Analysis of Attitude Determination Methods Using GPS Carrier Phase Measurements,” Math. Probl. Eng., vol. 2012, pp. 1-10, 2012, doi: 10.1155/2012/596396.

[14] P. Teunissen and S. Verhagen, "On the Foundation of the Popular Ratio Test for GNSS Ambiguity Resolution," in 17th International Technical Meeting of The Satellite Division of the Institute of Navigation, Long Beach, California, 2004.

[15] S. Carcanague, "Low-cost GPS/GLONASS precise positioning algorithm in constrained environment," $\mathrm{PhD}$ Thesis, Institut National Polytechnique de Toulouse-INPT, 2013.

[16] O. Julien, G. Lachapelle, and M. E. Cannon, "Galileo L1 Civil Receiver Tracking Loops' Architecture,” in 2007 IEEE International Symposium on Circuits and Systems, New Orleans, LA, USA, 2007, pp. 1737-1741, doi: 10.1109/ISCAS.2007.377930.

[17] Z. Dai, "MATLAB software for GPS cycle-slip processing," GPS Solut., vol. 16, pp. 267-272, Apr. 2012, doi: 10.1007/s10291-011-0249-1.

[18] H.-K. Lee, J. Wang, and C. Rizos, "Effective Cycle Slip Detection and Identification for High Precision GPS/INS Integrated Systems," J. Navig., vol. 56, no. 3, pp. 475-486, Sep. 2003, doi: 10.1017/S0373463303002443.

[19] G. Xu and Y. Xu, GPS: theory, algorithms and applications. Springer, 2016. 